\section{Production of Insect Juvenile Hormone by the Microsporidian Parasite Nosema}

WHEN microsporidian parasites of the genus Nosema infect insects, abnormal growth and metamorphosis are commonly observed. Heavily infected pupæ of saturniid Lepidoptera moult to adults which retain many pupal characters ${ }^{1}$. When flour beetles of the genus Tribolium are similarly infected, they appear larger than normal and die at or before pupation ${ }^{2}$. We have found that the larger size of such infected beetle larvæ is a result of more rapid growth and of supernumerary moults. All these effects resemble those which can be produced by injection of insect juvenile hormone or by implantation of corpora allata, the endocrine gland which produces this hormone ${ }^{3-6}$. Three hypotheses could explain this similarity of parasitic infection and endocrine activity: (1) The parasite might cause hypertrophy of, or hypersecretion by, the corpora allata of the host. (2) The parasite might cause other tissues of the host to produce juvenile hormone. (3) The parasites, themselves, might produce materials with juvenile hormone activity. We have carried out experiments by which we can decide among these alternatives.

Our source of parasites was a group of infected cultures of Tribolium castaneum, for which we are indebted to Prof. A. F. Bell, of the Population Genetics Institute, Purdue University. Parasites from this species were successfully transmitted to several insect orders, including the following species: Coleoptera, Tribolium confusum, and Tenebrio molitor; Blattaria, Periplaneta americana, Blaberus craniifera, and Byrsotria fumigata; Lepidoptera: Galleria mellonella, Bombyx mori, and Hyalophora (Platysamia) cecropia. In all these species the parasite induces more rapid growth of the nymph or larva and inhibits metamorphosis to the adult stage.

When Bombyx mori or any of the Blattaria were allatectomized as nymphs or larvæ the intermoult period was extended by $30-50$ per cent, and they moulted to premature adults. Following sham operations the intermoult period was also extended, but only by 15-40 per cent. When Nosema was implanted into allatectomized hosts the intermoult period increased 15-40 per cent, but the animals moulted to another nymphal or larval stage. These experiments rule out the first hypothesis since it is apparent that the effects of the parasite are expressed even in the absence of corpora allata and, in fact, the parasite substitutes for the endocrine gland.

The second hypothesis was ruled out by placing parasites in chambers attached to allatectomized hosts. In some experiments the chambers were in direct contact with the hæmocol of the host; in others, they were separated by a 'Millipore' filter with a pore size of $0.47 \mu$. This pore size is too small to permit the passage of the microsporidia. In no case did the tissues of the host show signs of infection or hypertrophy. Infective parasites were recovered from the chambers. In seven of ten such experiments the response of the host was consistent with production of juvenile hormone by the parasites. In sixty experiments in which the chambers contained tissues of insects free of parasites, a juvenilizing effect was never noted. These experiments show that juvenile hormone is not produced by non-endocrine host tissues.

Finally, $3 \mathrm{gm}$. of Nosema spores were isolated from $48 \mathrm{gm}$. of dried, infected Tribolium, and, by standard techniques $^{8}$ used for the purification of juvenile hormone, $11 \mathrm{mgm}$. of a yellow oil was recovered. This hormone preparation showed activity when assayed on Hyalophora cecropia ${ }^{9}$. An extract prepared in the identical fashion from uninfected Tribolium displayed no activity.

We believe that these experiments provide strong evidence that this parasite displays a new mode of parasite pathology; that is, production, in excessive amounts, of a substance normal to the host by a parasite. This substance then acts to upset the normal mechanisms of control of growth and differentiation. We know of no previous description of such a pathogenic action by a parasite.

These investigations were supported by the National Science Foundation $(G-11234)$ and the Purdue Research Foundation.

\section{F. M. Fisher, JUn.* \\ R. C. SANBORN}

Department of Biological Sciences, Purdue University,

West Lafayette, Indiana.

* Present address: Department of Biology, Rice University, Houston 1, Texas.

1 Finlayson, L. H., and Walters, V. Ann, Nature, 180, 713 (1957).

${ }^{2}$ West, A. F., J. Parasitol., 46, 747 (1960).

s Wigglesworth, V. B., Quart. J. Micro. Sci., 78, 91 (1936).

- Pfugfelder, O., $Z$. wiss. Zool., 152, 159 (1939).

- Pfeiffer, I. W., Trans. Conn. Acad. Arts Sci., 36, 489 (1945).

- Radtke, A., Naturwiss., 29, 451 (1942).

${ }^{7}$ Scharrer, B., Endocrinol., 38, 46 (1946).

${ }^{8}$ Gilbert, L. I., and Schneiderman, H. A., Trans. Amer. Micro. Soc., 79, 38 (1960)

- Williams, C. M., Nature, 178, 212 (1956).

\section{Changes in Transpiration induced by Ethyl Alcohol}

EтнуL alcohol was used as an auxiliary solvent in the preparation of auxin solutions for a series of transpiration experiments with plants in water culture when the roots were supplied with different auxins at varying concentrations. This method appears to be fairly common for auxin studies ${ }^{1,2}$. After weighing, the auxin is dissolved in a small quantity of ethyl alcohol, and the resulting solution is diluted with water or a nutrient solution to obtain the concentration desired. The small amounts of alcohol used are usually considered to be harmless; nevertheless, it has been shown that ethyl alcohol may have modifying effects in auxin experiments even at low concentrations ${ }^{3}$. In an attempt to determine whether this is also the case in transpiration experiments, some experiments were carried out with nutrient solution to which had been added varying amounts of ethyl alcohol.

The rate of transpiration was measured by means of the corona hygrometer ${ }^{4}$, which allows continuous recordings of the transpiration through the determination of the water vapour pressure $\left(p \mathrm{H}_{2} \mathrm{O}\right)$ in the air which has passed the plant chamber. The following transpiration conditions were used: illumination of about 10,000 lux from a photo lamp of the $B$-type at a distance of $45 \mathrm{~cm}$., the light having been filtered through $10 \mathrm{~cm}$. of cooled water; atmospheric air free of carbon dioxide of a relative humidity of 50 per cent and a temperature of $20^{\circ} \mathrm{C}$. being passed along the leaves at a rate of about $1.3 \mathrm{~m}$. $/ \mathrm{sec}$. When the plants (6-7 days old barley seedlings grown in water culture) had established constant transpiration under these conditions, the nutrient solution surrounding the roots was replaced by a mixture of ethyl alcohol and 